Artigo Original

\title{
Equilíbrio corporal em crianças e adolescentes asmáticos e não asmáticos
}

\author{
Marta Cristina Rodrigues da Silva ${ }^{1}$ \\ Sara Teresinha Corazza ${ }^{1}$ \\ Juliana Izabel Katzer ${ }^{2}$ \\ Carlos Bolli Mota ${ }^{1}$ \\ Juliana Côrrea Soares ${ }^{1}$ \\ ${ }^{1}$ Centro de Educação Física e Desportos, Universidade Federal de Santa Maria, RS, Brasil \\ ${ }^{2}$ Escola Superior de Educação Física, Universidade Federal de Pelotas, RS, Brasil
}

\begin{abstract}
Resumo: O objetivo foi analisar e comparar o equilíbrio corporal em crianças e adolescentes asmáticos e não asmáticos. Fizeram parte do grupo de estudos 24 sujeitos com idades de 7 a 14 anos divididos em dois grupos: grupo asmático e grupo controle. Para avaliação do equilíbrio corporal utilizou-se uma plataforma de força. Foram utilizadas as condições, olhos abertos e fechados com três tentativas aleatórias, com duração de 30 segundos cada uma. Os resultados apontaram diferença significativa entre os grupos, no teste de equilíbrio com olhos abertos apresentando maior amplitude de deslocamento na direção ânteroposterior (COPap) $(p=0,04)$, e médio lateral $(\mathrm{COPml})(p=0,02)$ no grupo asmático. Enquanto que no teste com olhos fechados a diferença foi significante apenas na amplitude de deslocamento ântero-posterior (COPap) $(p=0,02)$ e Área de Elipse $(p=0,03)$. Desse modo, a asma com suas limitações e consequências parece influenciar negativamente no equilíbrio corporal de seus portadores quando comparados com crianças sem a patologia e da mesma faixa etária.
\end{abstract}

Palavras-chave: Asma. Equilíbrio postural. Exercício físico.

\section{Balance in children and teenagers asthmatics and without asthma}

\begin{abstract}
The aim of this study was analyze and compare the balance in children and teenagers with and without asthma. Twenty-four subjects aging between 7 and 14 years old took part in this study divided into two groups: asthmatic group and control group. To evaluate the balance one force plate was used. Opened and closed eyes conditions were evaluated in three trials of 30 seconds each. The results showed significant differences between groups in opened eyes condition, with higher amplitude of displacement in the anteriorposterior direction (COPap) $(p=0,04)$, and medio-lateral direction (COPml) $(p=0,02)$ in the asthmatic group. Whereas in closed eyes condition the difference was significant only in the amplitude of displacement in the anterior-posterior direction (COPap) $(p=0,02)$ and Ellipse Area higher $(p=0,03)$ in the asthmatic group. Therefore, asthma with its limitations and consequences seems to have a negative effect on postural balance of their patients when compared with normal children of similar age.
\end{abstract}

Keywords: Asthma. Postural Balance. Exercise.

\section{Introdução}

A asma é uma doença inflamatória crônica das vias aéreas que causa obstrução no fluxo respiratório (GLOBAL INITIATIVE FOR ASTHMA, 2010), além disso, pode privar e limitar indivíduos de uma vida normal (TAKETOMI et al., 2005; BROCKMANN et al., 2007) ocasionando absenteísmo escolar (PEREIRA et al., 2009, BATEMAN, 2008).

Em alguns casos, a asma é reversível, espontaneamente ou por intermédio de tratamento e, pode ser desencadeada por uma série de estímulos, tais como alérgicos, irritantes, climáticos e pela prática inadequada de exercício físico (SOCIEDADE BRASILEIRA DE PNEUMOLOGIA E TISIOLOGIA, 2000). No entanto, estudos afirmam que o exercício bem orientado e controlado auxilia na redução dos sintomas da asma (CASSOL et al., 2004; SILVA et al., 2005; MOISÉS, 2006; REZENDE et al., 2008; MORAES et al., 2007; BETIO et al., 2007).

Entre os benefícios que o exercício regular ou a prática de Educação Física Escolar podem trazer para seu executante estão o desenvolvimento do equilíbrio corporal, tratandose da orientação mantida pela integração entre as informações sensoriais captadas pelos sistemas visual, vestibular, e somatossensorial, as atividades musculares e biomecânicas do corpo (HORAK E MACPHERSON, 1996; SHUMWAYCOOK e WOOLLACOTT, 2003). O equilíbrio corporal no asmático pode sofrer alterações em 
função de uma musculatura esquelética fraca, afetando as capacidades funcionais ( $\underline{\mathrm{ROCCO}}$, 2009) e também pela ansiedade que o indivíduo asmático enfrenta nas crises ( 2013).

Estas alterações podem ainda ser potencializadas pela associação de outros fatores como o uso de corticóides, a nutrição e a hipoxemia (AMERICAN THORACIC SOCIETY/ EUROPEAN RESPIRATORY SOCIETY, 1999; PASCHOAL e PEREIRA, 2001; DOURADO et al., 2006). Do mesmo modo, a manutenção da estabilidade corporal está relacionada ao balanceamento entre forças internas e externas, que agem no corpo durante a realização de ações motoras (MARTINS et al., 2010). Quando uma destas é afetada ou abalada por falta de estimulação, há um comprometimento do funcionamento corporal podendo ocasionar quedas, perda de força, utilização inadequada da musculatura (LUNARD et al., 2011) e diminuição no desempenho de várias atividades tanto desportivas quanto de vida diária (SOUZA, 2006; DUARTE et al., 2010). Ainda, os asmáticos apresentam maior sensação de dispnéia, o que determina uma interrupção precoce da atividade física e, por consequência, um estilo de vida mais sedentário (PIANOSI et al., 2004)

Essa instabilidade do equilíbrio corporal nos asmáticos tem sido comprovada através do estudo de Cunha et al. (2013) o qual através da plataforma de força, comparou o equilíbrio de adultos asmáticos e não asmáticos encontrando uma diferença na variável centro de pressão (COP), ou seja, uma maior amplitude de deslocamento para o COP nos sujeitos asmáticos. No entanto, Belli et al. (2009) através de registros fotográficos não encontraram diferença na postura em asmáticos e não asmáticos. Deste modo, para confirmar se há instabilidade corporal entre sujeitos asmáticos e não asmáticos, teve-se como objetivo analisar e comparar o equilíbrio corporal em crianças e adolescentes asmáticos e não asmáticos, praticantes de Educação Física Escolar.

\section{Metodologia}

\section{Grupo de estudo}

Fizeram parte do estudo 24 sujeitos, com idades entre 7 e 14 anos, divididos em dois grupos, grupo $1 \mathrm{com} 12$ sujeitos asmáticos, e grupo 2 com 12 sujeitos não asmáticos (grupo controle). Ambos os grupos de crianças e adolescentes participavam das aulas de Educação Física Escolar duas vezes por semana.

\section{Critérios de inclusão}

Foram adotados como critérios de inclusão para grupo asmático: possuir de 7 a 14 anos de idade, além do atestado médico comprovando a enfermidade, ser classificado como asmático (leve a moderado) através dos critérios do questionário de classificação da asma (IIII CONSENSO BRASILEIRO NO MANEJO DA ASMA, 2002), e também não ter nenhuma restrição ao exercício físico. Já para o grupo controle, não apresentar problemas respiratórios, não utilizar medicamentos de uso contínuo, não apresentar patologias correlacionadas que poderiam afetar o equilíbrio corporal.

\section{Local dos procedimentos}

O teste de equilíbrio foi realizado no laboratório de Ensino e Pesquisa do Movimento Humano do Centro de Educação Física e Desportos da Universidade Federal de Santa Maria (LAPEM/CEFD/UFSM), juntamente com obtenção das medidas da massa corporal e estatura dos sujeitos.

\section{Procedimentos éticos}

O presente estudo foi analisado e aprovado pelo Comitê de Ética da UFSM com CAAE número 0193.0.243.000-07. Os testes foram aplicados após assinatura do Termo de Consentimento Livre e Esclarecido pelos pais dos sujeitos da pesquisa.

\section{Instrumentos de avaliação}

Para a confirmação da enfermidade além do atestado médico utilizou-se o questionário de classificação da asma (III CONSENSO BRASILEIRO NO MANEJO DA ASMA, 2002), O qual foi aplicado individualmente $e$ de forma explicativa.

O equilíbrio corporal foi avaliado na plataforma de força, em condições bipodal, com olhos abertos e fechados, utilizando-se uma plataforma de força OR6-6-2000 AMTI (Advanced Mechanical Technology, INC), na qual os sujeitos foram instruídos a permanecer o mais estático possível, em cima da plataforma, na postura habitual, com os pés distanciados seguindo o alinhamento do quadril e com o olhar em um ponto fixo disposto a dois metros de distância do sujeito na altura dos olhos, conforme instruções de Teixeira et al. (2010). Foi demarcado o posicionamento dos pés em cima da plataforma 
para cada sujeito. Foram realizadas três tentativas de olhos abertos e três tentativas de olhos fechados de forma aleatória para cada sujeito, com 30 segundos cada, sendo a frequência de aquisição da plataforma de força de $100 \mathrm{~Hz}$. As informações provenientes da plataforma de força foram tratadas em uma rotina desenvolvida no ambiente Interactive Data Linguage (IDL). Primeiramente os dados foram filtrados utilizandose um filtro Butterworth passa-baixa de $4^{\underline{a}}$ ordem e frequência de corte de $10 \mathrm{~Hz}$. A seguir, os dados de força e momento foram utilizados no cálculo das duas coordenadas do centro de força (COP) a cada instante, uma na direção ânteroposterior e outra na direção médio-lateral, de acordo com o sistema de coordenadas da plataforma. Finalmente, a partir das coordenadas do COP, foram calculadas as variáveis de interesse. As variáveis analisadas foram a amplitude do centro de pressão nas direções ântero-posterior (COPap) e médio-lateral (COPml), Área de Elipse contendo 95\% dos dados do centro de pressão e velocidade de deslocamento do COP (COPvel).

Foram realizadas mensurações da estatura corporal, por meio de um estadiômetro da marca Welmy com resolução de $0,5 \mathrm{~cm}$ e da massa corporal, por meio de uma balança da marca Welmy com resolução de 0,05 kg.

\section{Análise estatística}

Realizou-se análise descritiva dos dados e após verificou-se a distribuição dos dados através do teste de Shapiro-Wilk. Comprovada a normalidade dos dados para ambos os grupos, utilizou-se a Anova para verificar se houve diferença nas variáveis analisadas, usando a idade como co-variável. Para isso fez-se uso do pacote estatístico SPSS for Windows, versão 14.0 com nível de significância de 5\%.

\section{Resultados}

$\mathrm{Na}$ Tabela 1 podem ser observadas as características do grupo de asmáticos e do grupo de não asmáticos.

Tabela 1. Análise descritiva dos grupos.

\begin{tabular}{ccc}
\hline VARIÁVEIS & $\begin{array}{c}\text { Asmáticos } \\
\text { média } \pm D P\end{array}$ & $\begin{array}{c}\text { Não Asmáticos } \\
\text { média } \pm D P\end{array}$ \\
\cline { 2 - 3 } Idade $(\mathrm{anos})$ & $9,75 \pm 2,34$ & $13 \pm 1,04$ \\
Massa $(\mathrm{kg})$ & $42,62 \pm 12,39$ & $54,10 \pm 8,9$ \\
Altura $(\mathrm{cm})$ & $1,39 \pm 0,11$ & $1,61 \pm 0,89$ \\
\hline
\end{tabular}

Em relação ao equilíbrio corporal, na comparação entre os dois grupos a Tabela 2 mostra que para o teste realizado com os olhos abertos, o grupo de asmáticos apresentou maior amplitude de deslocamento na direção ânteroposterior (COPap) e médio-lateral (COPml), do que o grupo de não asmáticos, sendo que a velocidade de deslocamento (COPvel) e a Área de Elipse não apresentaram diferença significativa entre os grupos. Já para o teste com olhos fechados a diferença foi significativa para a amplitude de deslocamento ântero-posterior (COPap) e para a Área de Elipse, sendo que os valores foram maiores para o grupo de asmáticos do que para o grupo de não asmáticos. Utilizou-se a idade como co-variável e essa não demonstrou influência significativa estatisticamente.

Tabela 2. Média dos parâmetros do equilíbrio corporal entre grupos em crianças e adolescentes asmáticos e não asmáticos.

\begin{tabular}{|c|c|c|c|c|}
\hline & \multicolumn{4}{|c|}{ GRUPOS } \\
\hline & $\begin{array}{c}\text { Asmáticos } \\
\text { média } \pm \mathrm{DP}\end{array}$ & $\begin{array}{l}\text { Não Asmáticos } \\
\text { média } \pm \text { DP }\end{array}$ & Valor de $F$ & Valor de $p$ \\
\hline \multicolumn{5}{|l|}{ Testes com olhos abertos } \\
\hline $\begin{array}{l}\text { Amplitude de deslocamento COPap }(\mathrm{cm}) \\
\text { Amplitude de deslocamento - COPml }(\mathrm{cm}) \\
\text { COPvel }(\mathrm{cm} / \mathrm{s}) \\
\text { Área de elipse } 95 \%\left(\mathrm{~cm}^{2}\right)\end{array}$ & $\begin{array}{l}2,99 \pm 0,84 \\
2,36 \pm 1,37 \\
1,39 \pm 0,44 \\
3,95 \pm 2,72 \\
\end{array}$ & $\begin{array}{l}2,30 \pm 0,73 \\
1,37 \pm 0,65 \\
1,37 \pm 0,48 \\
2,29 \pm 1,74 \\
\end{array}$ & $\begin{array}{l}5,03^{*} \\
6,45^{\star} \\
0,72 \\
2,64\end{array}$ & $\begin{array}{l}0,04 \\
0,02 \\
0,79 \\
0,12\end{array}$ \\
\hline \multicolumn{5}{|l|}{ Testes com olhos fechados } \\
\hline $\begin{array}{l}\text { Amplitude de deslocamento - COPap }(\mathrm{cm}) \\
\text { Amplitude de deslocamento - COPml }(\mathrm{cm}) \\
\text { COPvel }(\mathrm{cm} / \mathrm{s}) \\
\text { Área de elipse } 95 \%\left(\mathrm{~cm}^{2}\right)\end{array}$ & $\begin{array}{l}3,64 \pm 1,20 \\
2,17 \pm 1,01 \\
1,82 \pm 0,69 \\
5,29 \pm 3,46\end{array}$ & $\begin{array}{l}2,69 \pm 0,91 \\
1,98 \pm 1,23 \\
1,67 \pm 0,58 \\
3,15 \pm 2,27\end{array}$ & $\begin{array}{l}6,61^{*} \\
0,71 \\
0,571 \\
5,46^{*}\end{array}$ & $\begin{array}{l}0,02 \\
0,41 \\
0,57 \\
0,03\end{array}$ \\
\hline
\end{tabular}

*para $p<0,05$ 


\section{Discussão}

Partindo do pressuposto de avaliar e comparar o equilíbrio corporal em crianças e adolescentes asmáticos e não asmáticos, praticantes de Educação Física Escolar, encontraram-se algumas diferenças significantes na realização do teste com olhos fechados para os sujeitos asmáticos que apresentaram uma maior oscilação nas variáveis analisadas em comparação aos sujeitos não asmáticos. Teixeira et al. (2010) e Sousa et al. (2010) afirmam que quando ocorre uma alteração visual, proprioceptiva ou vestibular surgem alterações que caracterizam o desequilíbrio. $O$ equilíbrio corporal é mantido pela integração destes três componentes HORAK e MACPHERSON, 1996; SHUMWAY-COOK e WOOLLACOTT, 2003) e quando há falta de alguma destas informações percebe-se um comprometimento na estabilização corporal e a visão é um dos componentes importantes no equilíbrio postural. Sua remoção como no caso do teste com os asmáticos dificultou a obtenção de uma representação adequada do corpo no espaço. Estudos já demonstram a importância da visão para o equilíbrio postural como no estudo de Maggi et al. (2011) que realizaram estudos com um grupo de crianças cegas e indivíduos sem nenhum comprometimento visual, no qual relataram a diferença no equilíbrio corporal entre os grupos, demonstrando um déficit de equilíbrio com indivíduos cegos, ressaltando que indivíduos com características patológicas podem trazer comprometimentos para a estabilidade postural.

Essa dificuldade encontrada pelo sujeito asmático para manter o equilíbrio corporal faz com que muitos desses acabem privando-se da prática de atividades físicas contribuindo para a manutenção de um estilo de vida sedentário (PIANOSI et al., 2004) e que, por consequência pode afetar o seu desenvolvimento perceptivo motor.

Estudos afirmam que o exercício bem orientado e controlado auxilia também na redução dos sintomas da asma (CASSOL et al., 2004; SILVA et al., 2005; MOISÉS, 2006; REZENDE et al., 2008; MORAES et al., 2007; BETIO et al., 2007), desse modo com a sintomatologia controlada o indivíduo se torna mais participativo em atividades físicas contribuindo para uma melhora no seu desempenho físico e, principalmente na sua qualidade de vida. Estudos já demonstram que o exercício físico pode melhorar a condição de equilíbrio corporal (MANN et al., 2009) no qual apontam o quanto é importante o exercício físico para melhoria tanto dos sistemas sensoriais quanto do sistema motor e ainda, que a prática de exercícios físicos mostrou-se eficaz na redução das instabilidades posturais em diferentes populações.

A partir da verificação da instabilidade do equilíbrio corporal no grupo asmático, pode-se projetar um comprometimento do funcionamento corporal, em função de uma musculatura esquelética fraca (ROCCO, 2009) podendo ser um fator causador de quedas e também de déficit de desempenho em várias atividades tanto desportivas quanto de vida diária (SOUSA, 2010; DUARTE et al., 2010). A musculatura fraca juntamente com a postura corporal não alinhada pode comprometer o equilíbrio postural, o que é defendido por LUNARDI et al. (2011) quando descreve que o asmático tende a manter sua cabeça e ombros mais para frente, com um aumento interno da rotação do ombro, baixando a expansão da parede torácica e com isso se torna reduzida a flexibilidade da coluna vertebral torácica. Estas alterações podem causar um deslocamento do centro de gravidade do corpo e, portanto, afetar os resultados da posturografia. $E$ por isso uma maior velocidade de deslocamento e maior área do COP nas condições com a plataforma móvel, não podem ter sido capaz de alcançar a integração adequada de visão e informação vestibular. Entretanto, Belli et al.(2009) ao comparar asmáticos e não asmáticos através de registros fotográficos, não encontraram diferenças estatisticamente significativas.

Estudo de Cunha et al. (2013) corroboram com a presente investigação, revelando um déficit no equilíbrio corporal de adultos asmáticos, ao encontrar uma maior área de oscilação em asmáticos quando comparados com não asmáticos, mostrando que desde a infância, asmáticos apresentam um menor desempenho para o equilíbrio corporal.

Além disso, o sistema vestibular, quando afetado, atua como o principal responsável pelas vertigens e tonturas que causam os desequilíbrios corporais (SOARES, 2006), a oscilação na plataforma de força, demonstra e pode estar relacionada com a dificuldade que a criança com incapacidades motrizes possui de manter 0 equilíbrio corporal, em virtude de não praticar 
atividade física e também da patologia que limita a boa condição física de manter uma postura corporal ereta. Outro fator que influencia negativamente para a manutenção do equilíbrio corporal em asmáticos é o uso de corticóides. MOURA et al.(2002), em estudos longitudinais apontam para o uso contínuo de corticóide o motivo da atrofia muscular e conseqüentemente seu enfraquecimento, 0 que repercute negativamente no equilíbrio corporal.

Estudos com populações especiais, também comprovam a importância da estimulação de práticas corporais para desenvolver um bom equilíbrio, como Allegretti et al. (2007) que mostrou que o treino de equilíbrio promoveu a melhora do ajuste postural em ortostatismo, como também a melhora funcional do equilíbrio em crianças com paralisia cerebral com faixa etária de oito anos de idade. No estudo de Campos (2003) também houve melhora do equilíbrio corporal de crianças com perda auditiva após treinamento com mini-trampolim. Desse modo, a presente investigação vem ao encontro dos estudos expostos anteriormente, confirmando que asmáticos tanto em situação de olhos abertos quanto fechados apresentaram diferenças em algumas variáveis (Copap, Copml e Área de Elipse) quando comparados com o grupo assintomático, demonstrando comprometimentos com o equilíbrio corporal.

\section{Conclusão}

Por fim, através destes resultados afirma-se que a asma com suas limitações e consequências parece influenciar negativamente no equilíbrio corporal quando comparadas a crianças sem diagnóstico da patologia.

\section{Referências}

ALEGRETTI, K. M. G.; KANASHIRO, M. S.; MONTEIRO V. C.; BORGES H.C.; FONTES, S. V. Os efeitos do treino de equilíbrio em crianças com paralisia cerebral diparética espástica. Revista de Neurociências, São Paulo, v. 15, n. 2, p. 108113, 2007.

AMERICAN THORACIC SOCIETY/ EUROPEAN RESPIRATORY SOCIETY. Skeletal muscle dysfunction in chronic obstructive pulmonary disease. American Journal of Respiratory and Critical Care Medicine, New York, v. 159, p.1-40, 1999.

BATEMAN, E.; BOUSQUET, J., FITZGERALD, M.; HAAHTELA, T.; OHTA, K.; PAGGIARO P, et al. Global Strategy for Asthma Management and prevention. GINA, Cape Town, 2008.

BELLI, J. F.; CHAVES, T. C.; DE OLIVEIRA, A. S.; GROSSI, D. B. Analysis of body posture in children with mild to moderate asthma. European Journal of Pediatrics, Heidelberg, v. 168, n.1207, 2009.

BETIO, J.; KREBS, R. J.; KEULEN, G. V. Atividade física para portadores de asma. Cinergis, Santa Cruz do Sul, v. 8, n.2. p. 7-12, 2007.

BROCKMANN, P. V. et al. Actividad física y obesidad en ninõs com asma. Revista Chilena de Pediatria, Santiago, v. 78 , n. 5, p. 482-488, 2007.

CAMPOS, C. Efeitos de um programa de treinamento com trampolim acrobático sobre o equilíbrio de crianças surdas. Revista Sobama, São Paulo, v. 8, n. 1, p, 21-26, 2003.

CASSOL, V. E.; TREVISAN, M. E.; MORAES, E. Z.; PORTELA, L. O. C.; MENNA BARRETO, S.S. Broncoespasmo induzido pelo exercício em crianças e adolescentes com diagnóstico de asma. Jornal Brasileira de Pneumologia, Brasília, v. 30, n.2, p. 102-108, 2004.

CUNHA, Â. G. J.; NUNES,M. P. T.; RAMOS, R. T.; PINTO, R. M. C.; TANAKA, C.Balance Disturbances in Asthmatic Patients. Journal of Asthma. New York, v. 50, n. 3, p. 282-286. 2013.

DOURADO, V. Z.; TANNI, E. T.; VALE, A. S.; FAGANELLO, M. M; SANCHEZ, F,F.; GODOY, I. Manifestações sistêmicas na doença pulmonar obstrutiva crônica. Jornal Brasileiro de Pneumologia, Brasília, v. 32, n. 2, p. 161-171, 2006.

DUARTE, M; FREITAS, S. M. S. F. Revisão sobre posturografia baseada em plataforma de força para avaliação do equilíbrio; Revista Brasileira de Fisioterapia, São Carlos, v. 14, n. 3, p. 183192, 2010.

GLOBAL INITIATIVE FOR ASTHMA.. Global Strategy for Asthma Management and Prevention, GINA. Bethesda, v.38, sup.1, 2012. Disponível em:

http://www.ginasthma.org/pdf/GINA Report 2010. pdf. Acesso em 24 mai 2013.

HORAK, F.B.; MACPHERSON, J.M. Postural orientation and equilibrium, In: ROWELL, L.B.; SHERPHERD, J.T. (Ed.) Handbook of physiology: a critical, comprehensive presentation of physiological knowledge and concepts. New York: Oxford American Physiological Society. p. 255-92, 1996. 
III CONSENSO BRASILEIRO NO MANEJO DE ASMA. SOCIEDADE BRASILEIRA DE ALERGIA E IMUNOPATOLOGIA; SOCIEDADE BRASILEIRA DE PEDIATRIA; SOCIEDADE BRASILEIRA DE PNEUMOLOGIA E TISIOLOGIA. Jornal de Brasileiro de Pneumologia, Brasília, v.18, Supl. 1, p 1-28, 2002.

LUNARDI, A C.; MARQUES, S. C. C.; RODRIGUES, M.. FA.; MARQUES, A.P. STELMACH, R.; FERNANDES, C. C.R. Musculoskeletal dysfunction and pain in adults with asthma. Journal Asthma. New York, v. 48, n.1, p. 105-110, 2011. doi: http://dx.doi.org/10.3109/02770903.2010.520229 pp.105-10.

MAGGI, A. B.; SOUZA, V. R.; SINÉSIO, T; MACHADO, D.S.; VIEIRA, M. M. A influência da visão no equilíbrio de crianças deficientes visuais congênitas e com visão normal.

http://www.efdeportes.com/ EFDeportes.com, Revista Digital EFDeportes. Buenos Aires, Año 15, № 154, Marzo de 2011.

MANN, L; KLEINPAUL, J. F; MOTA, C. B; SANTOS, S. G. Equilíbrio corporal e exercícios físicos: uma revisão sistemática. Motriz- Revista de Educação Física - Unesp, Rio Claro, v.15, n.3, p.713-722, 2009.

MARTINS, A.; PEREIRA, E. F.; TEIXEIRA, C. S. I.; CORAZZA, S.T. Relação entre força dinâmica máxima de membros inferiores e o equilíbrio corporal em praticantes de musculação. Revista Brasileira de Cineantropometria \& Desempenho Humano, Florianópolis, v. 12, n. 5, p. 375-380, 2010.

MOISÉS, M. P. Ginástica respiratória para asmáticos: efeito de redução do número e intensidade de crises asmáticas. Revista Mackenzie de Educação Física e Esporte, São Paulo, v. 5, p. 76-81, 2006.

MORAES, G. M. L.; NOVO, N. F.; JULIANO, Y.; CURY, M. C. F. S.; BOGOSSIAN, M.

Comportamento do fluxo expiratório antes e após aula de natação em crianças portadoras de asma. Revista Sociedade Brasileira Clínica de Medicina. São Paulo, v. 5, n.1, p. 7-13, 2007.

MOURA, J. A. R.; CAMARGOS, P. A. M.; BLIC, J. Tratamento profilático da asmaProphylactic treatment of asthma. Jornal de Pediatria, Rio de Janeiro, v. 78, n.2, p. 141-150, 2002. Disponível em:

http://www.scielo.br/pdf/jped/v78s2/v78n8a05.pdf. Acesso em 24 mai 2013.
PASCHOAL, I. A.; PEREIRA, M. C. Efeitos Sistêmicos da DPOC. Livro de Atualização em Pneumologia. Revinter; 2001.

PEREIRA, E. F.; TEIXEIRA, C. S.; VILLIS, J. M. C.; PAIM, M. C. C.; SANCHOTENE, E. L. D. Fatores motivacionais de crianças e adolescentes asmáticos para a prática da natação; Revista Brasileira de Ciência \& Movimento, São Paulo, v. 17, n. 3, p. 9-17, 2009. Disponível em: http://portalrevistas.ucb.br/index.php/RBCM/article /viewFile/1037/1399. Acesso em 24 mai 2013.

PIANOSI, P. T.; DAVIS, H. S. Determinants of physical fitness in children with asthma.

Pediatrics. v. 113, n.3, p. 225-229, 2004.

REZENDE, I. M. O. et al. Efeitos da reabilitação pulmonar sobre a qualidade de vida: uma visão das crianças asmáticas e de seus pais. Acta Fisiátrica, São Paulo, v.15, n.3, p. 165-169, 2008.

ROCCO, C. C. M. Aspectos Neurofisiológicos e sua Relação com o Acometimento Clínico e Funcional dos Pacientes com Doença Pulmonar Obstrutiva Crônica. Dissertação (Mestrado em Ciências da Reabilitação) Programa de Pós-Graduação, Universidade Nove de Julho; São Paulo, 2009.

SCHUMWAY-COOK A.; WOOLLACOTT MH. Controle postural. In: Schumway-Cook A, Woollacott MH. Controle Motor: teoria e aplicações práticas. 2a ed. Barueri: Manole; p.153-178, 2003.

SILVA, C. S.; TORRES, L. A. G. M. M.; RAHAL, A.; FILHO, J. T.; VIANNA, E. O. Avaliação de um programa de treinamento físico por quatro meses para crianças asmáticas. Jornal Brasileiro de Pneumologia, Brasília, v.31, n.4, p. 270-285, 2005.

SOARES, E. V. Reabilitação vestibular em idosos com desequilíbrios para marcha. Rev.

Perspectivas Online, v. 6, p. 88-100, 2006.

SOUSA, A. M. M.; BARROS, J. F.; NETO, B. M. S.; Gorla, J. I. Avaliação do controle postural e do equilíbrio em crianças com deficiência auditiva. Revista da Educação Física/UEM, Maringá, v. 21 , n. 1, p. 47-57, 2010.

SOUZA, C. M.; OLIVEIRA, C. W. F.; FERREIRA, A. C. G. O.; ALMEIDA, J. J. G. Ginástica Artística para crianças deficientes visuais. Relato de experiência. Revista Digital EFDeportes, Buenos Aires, v. 10, n. 94, p. 1-16, 2006.

TAKETOMI, E. A.; MARRA, S. M. G; Silva Segundo, GR. Fisioterapia em asma: Efeito na função pulmonar e em parâmetros imunológicos. 
Fitness and Performance Journal, Rio de Janeiro, RJ, v. 4, n.2, p. 97-100, 2005.

TEIXEIRA, C. S.; LEMOS, L. F. C.; LOPES L. F. D.; MOTA, C. B. A influência dos sistemas sensoriais na plataforma de força: estudo do equilíbrio corporal em idosas com e sem queixa de tontura. Revista CEFAC, São Paulo, v. 2, n. 6 , p. 1025-1032, 2010.

\section{Endereço:}

Marta Cristina Rodrigues da Silva

UFSM - Centro de Educação Física e Desportos

Av. Roraima, 1000 Campus UFSM

Santa Maria RS Brasil

97105970

Telefone: (55) 9146.6199

e-mail: martacrys1605@gmail.com

Recebido em: 25 de março de 2012.

Aceito em: 15 de maio de 2013.

\section{(c) (7)}

Motriz. Revista de Educação Física. UNESP, Rio Claro, SP, Brasil - elSSN: 1980-6574 - está licenciada sob Creative Commons - Atribuição 3.0 